\title{
Advances in polyelectrolyte multilayer nanofilms as tunable drug delivery systems
}

This article was published in the following Dove Press journal:

Nanotechnology, Science and Applications

5 August 2009

Number of times this article has been viewed

\section{Bingbing Jiang' \\ John B Barnett ${ }^{2,3}$ \\ Bingyun $\mathrm{Li}^{1,4,5 \#}$}

'Biomaterials, Bioengineering and Nanotechnology Laboratory, Department of Orthopaedics, School of Medicine, ${ }^{2}$ Department of Microbiology, Immunology, and Cell Biology, ${ }^{3}$ Center for Immunopathology and Microbial Pathogenesis, School of Medicine, ${ }^{5}$ Department of Chemical Engineering, College of Engineering and Mineral Resources, West Virginia University, Morgantown, WV, USA; ${ }^{4} \mathrm{WVNano}$ Initiative, Morgantown, WV, USA; "Aided by a grant from Osteosynthesis and Trauma Care (OTC) Foundation
Correspondence: Bingyun Li Director, Biomaterials, Bioengineering and Nanotechnology Laboratory, Drug Development and Pharmaceutics Study Group and WVNano Initiative, Department of Orthopaedics, School of Medicine, West Virginia University, Morgantown, WV 26506-9196, USA

Tel +l 3042931075

Fax +I 3042937070

Email bli@hsc.wvu.edu http://www.hsc.wvu.edu/som/ortho/ nanomedica-group/

\begin{abstract}
There has been considerable interest in polyelectrolyte multilayer nanofilms, which have a variety of applications ranging from optical and electrochemical materials to biomedical devices. Polyelectrolyte multilayer nanofilms are constructed from aqueous solutions using electrostatic layer-by-layer self-assembly of oppositely-charged polyelectrolytes on a solid substrate. Multifunctional polyelectrolyte multilayer nanofilms have been studied using charged dyes, metal and inorganic nanoparticles, DNA, proteins, and viruses. In the past few years, there has been increasing attention to developing polyelectrolyte multilayer nanofilms as drug delivery vehicles. In this mini-review, we present recent developments in polyelectrolyte multilayer nanofilms with tunable drug delivery properties, with particular emphasis on the strategies in tuning the loading and release of drugs in polyelectrolyte multilayer nanofilms as well as their applications.
\end{abstract}

Keywords: nanofilm, polyelectrolyte multilayer, drug delivery, electrostatic layer-by-layer self-assembly, biomedical device, surface modification

\section{Introduction}

Polyelectrolyte multilayer nanofilms are typically prepared using electrostatic layer-by-layer self-assembly (LbL), which has been widely used as a versatile technique for fabricating nanofilms with controlled structures and compositions. ${ }^{1}$ Due to the high degree of control over film properties, flexible choice of assembly components, and ease of processing, polyelectrolyte multilayer nanofilms have a variety of applications ranging from optical and electrochemical materials to biomedical devices..$^{2-4}$ Most studies to date have focused on engineering the specifics of polyelectrolyte multilayer nanofilms in terms of efficiency of ion-pairing, internal thin film architecture, ion permeability, porosity, wetting/dewetting properties, and surface roughness as well as mechanical properties. The biocompatibility of polyelectrolyte multilayer nanofilms has also been investigated. In vitro and in vivo studies have shown that some polyelectrolyte multilayer nanofilms are biocompatible, and may enhance adhesion and growth of cells like osteoblasts, ${ }^{5,6}$ chondrocytes,,${ }^{7,8}$ myoblasts, ${ }^{9}$ chondrosarcomas, ${ }^{10}$ and smooth muscle cells. ${ }^{11}$

Over the past few years, there has been increasing interest in developing drug-carrying polyelectrolyte multilayer nanofilms for biomedical applications, which may offer the ability to control the structure and concentration of incorporated drugs in precise-scale. A variety of drugs such as proteins and DNA have been incorporated into polyelectrolyte multilayer nanofilms (Table 1), retained their bioactivity, ${ }^{12,13}$ and showed promise in treating diseases including biomedical 
device-associated infection. ${ }^{7,14-18}$ Here we briefly review the research progress in polyelectrolyte multilayer nanofilms as tunable drug delivery systems and their applications.

\section{Tunable drug loading and release in polyelectrolyte multilayer nanofilms}

Polyelectrolyte multilayer nanofilms are prepared using LbL nanotechnology, which is currently one of the most powerful methods for preparing nanofilms of controlled molecular architecture. LbL technology, developed in the $1990 \mathrm{~s},{ }^{1}$ involves the repetitive sequential dipping of a charged substrate into solutions of oppositely-charged polyelectrolytes, and the adsorption of the oppositelycharged polyelectrolytes leads to the formation of a multilayer nanofilm on the substrate, eg, a device (Figure 1). Compared to other coating techniques, such as the plasma technique, LbL provides a rather simple aqueous-based means of varying film composition while providing enormous design flexibility. The properties of polyelectrolyte multilayer nanofilms can easily be manipulated. ${ }^{19}$ The physical basis of $\mathrm{LbL}$ is primarily electrostatic attraction; however, other forces, eg, hydrophobic, van der Waals, and hydrogen bonding or acid-base type, may also play a significant role under certain conditions. These forces can be used to control drug loading and release from polyelectrolyte multilayer nanofilms.

\section{Tunable drug loading in polyelectrolyte multilayer nanofilms}

In the last decade, polyelectrolyte multilayer nanofilms have attracted great interest as potential vehicles for controlled drug delivery. In general, drugs can be incorporated within polyelectrolyte multilayer nanofilms through a variety of means including $\mathrm{pH}$-induced drug loading, integrating drugs as an LbL-coating component, or covalent bonding of the drug to nanofilms.

We recently reported the loading of small charged drug molecules (eg, cefazolin and gentamicin) into polypeptide multilayer nanofilms, and we found that the loading of small charged drug molecules could be finely tuned by the number of film layers, drug incubation time, and application of heat treatment after film formation. ${ }^{17}$ Moreover, we showed that drug loading was $\mathrm{pH}$-dependent and could be tuned by drug-loading solution $\mathrm{pH}$ as well as film preparation $\mathrm{pH}$.

Table I Examples of polyelectrolyte multilayer nanofilms as tunable drug delivery systems

\begin{tabular}{|c|c|c|c|c|c|}
\hline $\begin{array}{l}\text { Polyelectrolyte } \\
\text { multilayer nanofilms }\end{array}$ & Drug & $\begin{array}{l}\text { Drug loading } \\
\text { mechanism }\end{array}$ & $\begin{array}{l}\text { Drug release } \\
\text { mechanism }\end{array}$ & Applications & References \\
\hline$(\mathrm{PAH} / \mathrm{PAA})_{10.5}$ & Methylene blue & $\mathrm{pH}$-induced & $\mathrm{pH}$-induced & Controlled drug delivery & Chung et al. ${ }^{32}$ \\
\hline$(\text { PLL/PLGA })_{20}$ & Cefazolin, gentamicin & $\mathrm{pH}$-induced & $\mathrm{pH}$-induced & Infection prevention & Jiang and $\mathrm{Li}^{17}$ \\
\hline $\begin{array}{l}\text { (PQ4VP-b-PtBA } \\
\text { /PAAbP4VP), }\end{array}$ & Pyrene, Nile Red & Charge-induced & Charge-induced & $\begin{array}{l}\text { Drug delivery of } \\
\text { hydrophobic molecules }\end{array}$ & Qi et al. ${ }^{20}$ \\
\hline$(\mathrm{PAH}-\mathrm{DEX} / \mathrm{PSS})_{10}$ & $\begin{array}{l}\text { Fluorescein, CdTe } \\
\text { nanoparticles }\end{array}$ & Charge-induced & Charge-induced & Controlled drug release & Wang et al. ${ }^{28}$ \\
\hline$(\mathrm{PNIPAAm} / \mathrm{PAA})_{10}$ & Rhodamine B & Temperature-induced & Temperature-induced & Stimulated drug delivery & Quinn et al. ${ }^{36}$ \\
\hline$(\mathrm{PSMA} / \mathrm{PEO})_{10}$ & Rhodamine B & Temperature-induced & Temperature-induced & Stimulated drug delivery & Quinn et al. ${ }^{22}$ \\
\hline $\begin{array}{l}(\mathrm{PAA} / \mathrm{PEO}-\mathrm{b}-\mathrm{PCL})_{30} \\
(\mathrm{PAA} / \mathrm{PEO}-\mathrm{b}-\mathrm{PCL})_{60}\end{array}$ & Triclosan & $\begin{array}{l}\text { Hydrophilic/hydrophobic } \\
\text { interaction }\end{array}$ & Degradation & $\begin{array}{l}\text { Drug delivery of } \\
\text { hydrophobic agents }\end{array}$ & Kim et al. ${ }^{37}$ \\
\hline $\begin{array}{l}{[\text { poly }(\beta \text {-amino ester }) /} \\
\text { heparin }]_{20}\end{array}$ & Heparin & As a coating component & $\begin{array}{l}\mathrm{pH} \text {-induced, } \\
\text { degradation }\end{array}$ & Controlled drug release & Wood et al. ${ }^{33}$ \\
\hline$(\mathrm{PPE} / \mathrm{pEGFP})_{10}$ & Plasmid DNA & As a coating component & Degradation & Prolonged gene delivery & Lu et al. ${ }^{30}$ \\
\hline$(\mathrm{PDDA} / \mathrm{DNase} \mathrm{I})_{8}$ & $\begin{array}{l}\text { DNA (complexed } \\
\text { with PDDA) }\end{array}$ & As a coating component & $\begin{array}{l}\text { Enzymatic } \\
\text { degradation }\end{array}$ & Prolonged gene delivery & Serizawa et al. ${ }^{38}$ \\
\hline$(\mathrm{HA} / \mathrm{CHI})_{6}$ & $\begin{array}{l}\text { Paclitaxel (covalently } \\
\text { bonded to HA) }\end{array}$ & As a coating component & Hydrolysis & $\begin{array}{l}\text { Treatment of breast/ } \\
\text { ovarian cancers }\end{array}$ & Thierry et al. ${ }^{12}$ \\
\hline$(\mathrm{PI} / \mathrm{P} 2)_{30}$ & $\begin{array}{l}\text { DTNB (covalently } \\
\text { bonded to PI) }\end{array}$ & $\begin{array}{l}\text { As a coating component, } \\
\mathrm{pH} \text {-induced }\end{array}$ & Redox-stimulated & Stimulated drug delivery & Zhong et al. ${ }^{21}$ \\
\hline
\end{tabular}

Abbreviations: PAH, poly(allylamine hydrochloride); PAA, poly(acrylic acid); PLL, poly(L-lysine); PLGA, poly(L-glutamic acid); PQ4VP-b-PtBA, quaternized poly(4-vinylpyridine)b-poly(tert-butylacrylate); PAAbP4VP, poly(acrylic acid)-b-poly(4-vinylpyridine); PAH-DEX, cross-linked PAH and dextran; PSS, poly(styrene sulfonate); PNIPAAm, poly(N-isopropylacrylamide); PSMA, poly(styrene-alt-maleic acid); PEO, poly(ethylene oxide); PEO-b-PCL, poly(ethylene oxide)-block-poly( $(\varepsilon$-caprolactone); PDDA, poly(diallyldim ethylammonium chloride); DNase I, endonuclease deoxyribonuclease I; HA, hyaluronan; $\mathrm{CHI}$, chitosan; PI, (KVKGKCKV) ${ }_{3}$ KVKGKCKY polypeptide; P2, (EVEGECEV) ${ }_{3}$ EVEGECEY polypeptide; DTNB, 5,5'-dithiobis(2-nitrobenzoic acid); PPE, poly(2-aminoethyl propylene phosphate); pEGFP, plasmid DNA encoding for enhanced green fluorescent protein. 

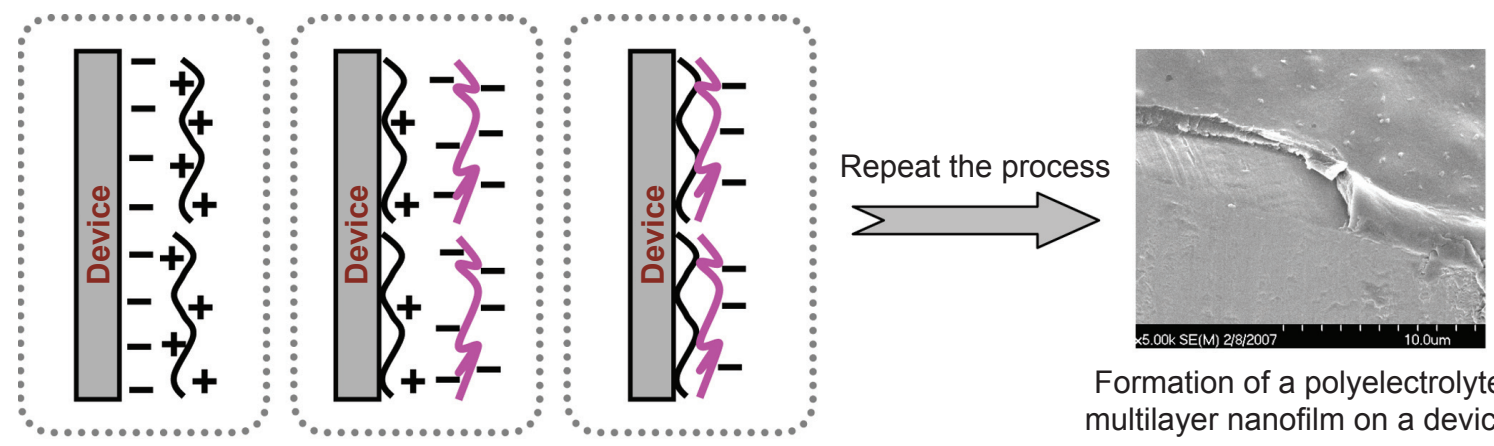

Formation of a polyelectrolyte multilayer nanofilm on a device

Figure I Formation of a polyelectrolyte multilayer nanofilm on a device using electrostatic layer-by-layer self-assembly

We created "binding-sites" or net charges within multilayer nanofilms for binding of oppositely-charged drug molecules by controlling the $\mathrm{LbL}$ process $\mathrm{pH}$ and the drug-loading solution $\mathrm{pH}$. As a result, we achieved tunable loading of antibiotics in polypeptide multilayer nanofilms. ${ }^{17}$ More recently, we found that the $\mathrm{pH}$ (in the range of 4.0-10.0) of the LbL process also influenced the secondary structure of polypeptide multilayer nanofilms. We observed more $\beta$-sheet structure in poly(L-lysine)/poly(L-glutamic acid) or PLL/PLGA multilayer nanofilms prepared at a lower (eg, $\mathrm{pH} 4.0$ ) or higher $\mathrm{pH}$ (eg, $\mathrm{pH} 10.0$ ) than at $\mathrm{pH} 7.0$ (Figure 2). The secondary structure of nanofilms may play a role in tuning drug loading as well as release in polyelectrolyte multilayer nanofilms although no such evidence has been reported yet. In other studies, amphiphilic block copolymer micelles, ${ }^{20,21}$ hydrogel, ${ }^{22,23}$ and nanostructured pores ${ }^{24}$ were also introduced in polyelectrolyte multilayer nanofilms to control drug-loading and release.

Moreover, by integrating drug molecules as an LbL coating component, a variety of charged macromolecular drugs, eg, proteins, polysaccharides, enzymes, nucleic acids, and DNA, have been immobilized into polyelectrolyte multilayer nanofilms. ${ }^{25-29}$ In these studies, the macromolecular drug molecules served as both functional drugs and film components. As a coating component, interleukin-12 p70 (IL-12 p70; a key cytokine for cell-mediated immune response), together with a protein carrier (ie, bovine serum albumin), was recently incorporated in polypeptide multilayer nanofilms and it was found that its loading and release were tunable by controlling the number of film layers as well as IL-12 p70 concentration. $^{18}$

In addition, drug molecules can be covalently bonded to polyelectrolyte multilayer nanofilms. ${ }^{12,30,31}$ A drug delivery platform in hyaluronan/chitosan or HA/CHI multilayer nanofilms was developed. Paclitaxel, a chemotherapeutic agent, was conjugated on the HA chain within the nanofilms, and its drug bioactivity was found to be retained. ${ }^{12}$ Werner and colleagues ${ }^{31}$ coupled an adhesion peptide, derived from laminin, in PLL/PLGA multilayer nanofilms, and observed enhanced epithelial cell adhesion and better in vivo cell colonization.

\section{Tunable drug release from polyelectrolyte multilayer nanofilms}

Drug release behavior in polyelectrolyte multilayer nanofilms depends on the permeability, erosion or degradation of multilayer nanostructure, as well as the interaction between drug molecules and nanofilm components. As a result, the release of drugs can be tuned by control of environment $\mathrm{pH}$, ionic strength, temperature, and deconstruction or dissolution of polyelectrolyte multilayer nanofilms.

Our studies showed that environment $\mathrm{pH}$ could be used to tune the release of electrostatically-bound drug molecules, eg, cefazolin and gentamicin, and the $\mathrm{pH}$-triggered drug release was due to the change of net charges of weak polyelectrolytes within the nanofilms as $\mathrm{pH}$ changes. ${ }^{17}$ The change in net charges results in attraction or repulsion between charged drug molecules and polyelectrolyte film components thereby leading to the tuning of drug release. Polyelectrolyte multilayer nanofilms composed of poly(allylamine hydrochloride)/ poly(acrylic acid) also had pH-responsive release of small charged drug molecules. ${ }^{32}$ In addition, drug release could be controlled by introducing $\mathrm{pH}$-degradable components in polyelectrolyte multilayer nanofilms, as $\mathrm{pH}$-induced degradation and drug release were observed in multilayer nanofilms assembled with degradable poly( $\beta$-amino ester $).^{33}$

Similarly, ionic strength can be utilized to tune drug release, since changing the ionic strength of the drug release medium can influence the electrostatic interactions between polyelectrolyte coating components and charged drug molecules. It was found that high concentrations of salt (eg, $0.6 \mathrm{M}$ ) weakened the electrostatic interactions between 


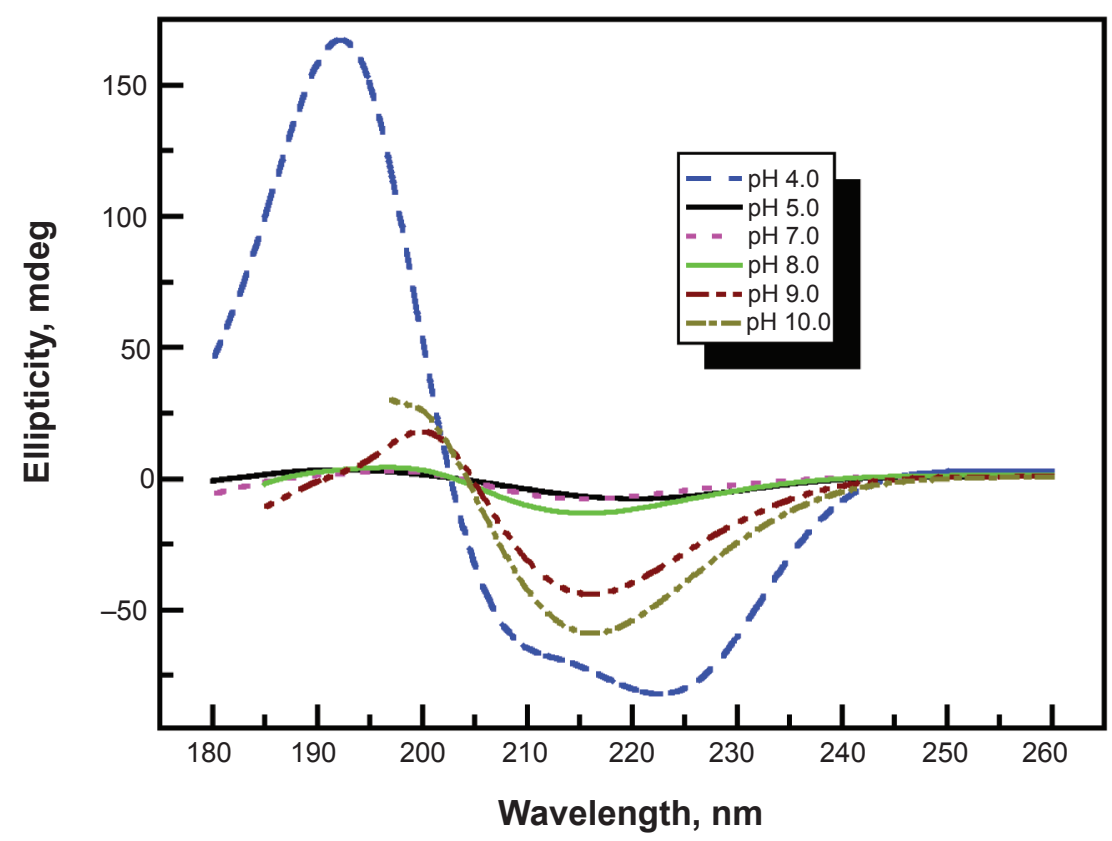

Figure 2 Effects of $\mathrm{pH}$ at which nanofilms were prepared on secondary structure of (PLL/PLGA) 10 nanofilms. (PLL/PLGA) 10 nanofilms were assembled on quartz slides at $\mathrm{pHs} 4.0,5.0,7.0,8.0,9.0$, and 10.0 .

adjacent layers of polyelectrolytes and caused the destruction of polyelectrolyte multilayer nanofilms. ${ }^{34,35}$

Temperature-triggered drug release is another attractive approach to tune drug release from polyelectrolyte multilayer nanofilms. Polyelectrolyte multilayer nanofilms were constructed by introducing a thermo-responsive hydrogel, eg, poly(N-isopropylacrylamide), as a drug delivery vehicle. ${ }^{36}$ The poly(N-isopropylacrylamide) hydrogel undergoes a reversible volume phase transition at its critical point temperature, at which it shrinks or expands. This reversible shrinking/expanding structure therefore leads to tunable drug-loading and release. Quinn and colleagues also studied polyelectrolyte multilayer nanofilms assembled with poly(styrene-alt-maleic acid) and poly(ethylene oxide); the latter is thermo-responsive. ${ }^{36}$ Rhodamine $\mathrm{B}$, a commonlyused dye, was used as a drug model and loaded into the nanofilms. It was found that the release of Rhodamine B was temperature-dependent. ${ }^{36}$

Finally, introducing crosslinking, hydrolysis, or enzymatic degradation can tune drug release from polyelectrolyte multilayer nanofilms. Amphiphilic block copolymer micelles were integrated as nanometer-sized vehicles for hydrophobic drug delivery, and sustained release of triclosan was obtained from polyelectrolyte multilayer nanofilms after introducing crosslinking. ${ }^{37}$ Hydrolysis of the ester linkage between HA and paclitaxel under physiological conditions also showed controlled release of paclitaxel from $\mathrm{HA} / \mathrm{CHI}$ multilayer nanofilms. ${ }^{12}$ Tunable release of procoagulant agents and DNA was observed in enzyme hydrolysable polyelectrolyte multilayer nanofilms. ${ }^{38,39}$

\section{Applications of polyelectrolyte multilayer nanofilms as tunable drug delivery systems}

Polyelectrolyte multilayer nanofilms have been widely used to modify the surface properties of biomedical devices. Drug-carrying polyelectrolyte multilayer nanofilms have recently attracted attention for their antibacterial applications, inflammation alleviation, and tissue engineering, to name a few examples.

The surface of biomedical devices is a common site of bacterial and fungal adhesion, the first step in the formation of a biofilm that frequently leads to infections. In order to prevent such infections, several physical and chemical modifications of device surface have been studied, and the LbL process is one promising technique. Our studies showed that PLL/PLGA multilayer nanofilms with tunable antibiotic loading prevented the colonization of Staphylococcus aureus on orthopedic implants and demonstrated controllable antibacterial properties in vitro against S. aureus. ${ }^{17}$ Cefazolin and gentamicin-loaded PLL/PLGA nanofilms with different numbers of layers and prepared at different solution $\mathrm{pHs}$ showed different antibacterial 
properties. Also, our developed IL-12 p70 incorporated polypeptide multilayer nanofilm proved its efficacy in preventing biomedical device-associated infection; IL-12 p70 on stainless steel Kirschner wires substantially decreased bacterial infection in an open femur fracture rat model. ${ }^{18}$ Also, CHI, a commonly used antibacterial dressing, has received attention in fabrication of antibacterial polyelectrolyte multilayer nanofilms. CHI-containing multilayer nanofilms showed high resistance to bacterial adhesion and led to a substantial decrease in Escherichia coli adhesion compared to control bare substrates. ${ }^{7,14,15}$ Moreover, CHIcontaining multilayer nanofilms prepared at different ionic strengths showed different bacterial resistance properties, ${ }^{7}$ and the $\mathrm{LbL}$ process solution $\mathrm{pH}$ had a remarkable effect on the antibacterial properties of polyelectrolyte multilayer nanofilms. ${ }^{14}$ Antibacterial nanoparticles such as silver nanoparticles could also be immobilized in polyelectrolyte multilayer nanofilms and enabled the nanofilms antibacterial properties against methicillin-resistant $S$. aureus or MRSA. ${ }^{16}$ In addition, antifungal polyelectrolyte multilayer nanofilms were developed by incorporating chromogranin A-derived antifungal peptide in PLL/PLGA multilayer nanofilms, and in vitro studies demonstrated the ability of the nanofilms in inhibiting growth of the yeast Candida albicans and the filamentous fungus Neurospora crassa..$^{40}$

Polyelectrolyte multilayer nanofilms incorporated with drugs may also alleviate inflammation associated with biomedical devices. Schultz and colleagues ${ }^{29}$ conjugated a synthetic analog of an anti-inflammatory peptide, alphamelanocyte-stimulating hormone (alpha-MSH) to PLGA, and their developed PLL/PLGA multilayer nanofilms showed reduced inflammatory response in vivo.

Further, polyelectrolyte multilayer nanofilms have been investigated to functionalize biomedical device surfaces for tissue engineering applications. Controlled delivery of growth factors and cytokines from biomedical device surfaces may offer the potential to concentrate the drugs and deliver them locally, as opposed to topical administration. Immobilization of growth factors and cytokines within polyelectrolyte multilayer nanofilms may also protect the drugs from degradation by enzymes in tissue fluids. Localized and sustained drug delivery systems were designed for recombinant human bone morphogenetic-protein 2 (rhBMP-2) in polyelectrolyte multilayer nanofilms. ${ }^{13}$ Crosslinked PLL/HA multilayer nanofilms were prepared as reservoirs for rhBMP-2 delivery to myoblasts and induced their differentiation into osteoblasts in a dose-dependent manner. The amount of rhBMP-2 loaded in the nanofilms was controlled by varying the deposition conditions and the film thickness, and the immobilized growth factor was found to be bioactive. Such polyelectrolyte multilayer nanofilms could have potential applications in local delivery of growth factors for tissue engineering constructs.

\section{Conclusions and outlook}

Polyelectrolyte multilayer nanofilms with tunable drug delivery have attracted great interest and shown promise for the development of coatings for implant devices and tissue engineering scaffolds. A variety of drugs including antibiotics, cytokines, and growth factors have been incorporated in polyelectrolyte multilayer nanofilms. The incorporation and release of drugs can be finely tuned by environmental conditions, including $\mathrm{pH}$, temperature, and ionic strength; by the history of nanofilm preparation, including physical binding or chemical bonding of drug molecules within polyelectrolyte multilayer nanofilms, and $\mathrm{pH}$, concentration, and ionic strength of polyelectrolyte self-assembly solutions and drug-loading solutions; as well as by post-preparation treatments (eg, heat treatment).

Future development in this area could be polyelectrolyte multilayer nanofilms possessing multifunctions including combined delivery of multiple therapeutic agents or combined delivery of therapeutic and diagnostic agents. Multiple therapeutic and/or diagnostic agents could be immobilized on the surface of one biomedical device (eg, an orthopedic implant) via formation of polyelectrolyte multilayer nanofilms, and different drug-loading strategies described in this paper could be used to incorporate multiple drugs in polyelectrolyte multilayer nanofilms (Figure 3a). Depending on the means that drugs are incorporated, multiple drug delivery patterns, eg, burst release, sustained release, and constant concentration, could be obtained in one device (Figure 3b). Such devices could achieve ideal treatment outcomes, eg, achieving infection prevention and wound healing simultaneously or combining diagnosis and treatment in a single device.

\section{Acknowledgments}

Financial support from National Science Foundation (OISE0737735), AO Foundation, Osteosynthesis and Trauma Care Foundation, National Aeronautics and Space Administration West Virginia Experimental Program to Stimulate Competitive Research (NASA WV EPSCoR), and West Virginia University Research Corporation, is acknowledged. Project S-07-43L was supported by the AO Research Fund of the AO Foundation. Any opinions, findings, and conclusions or 
a

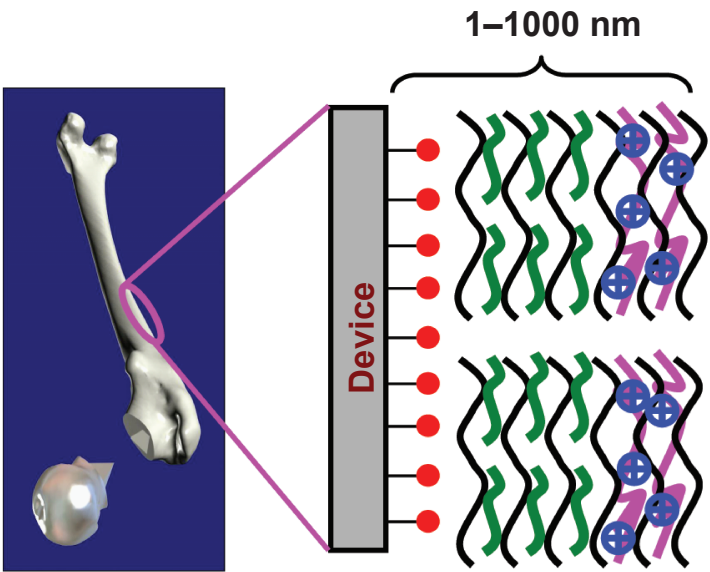

b

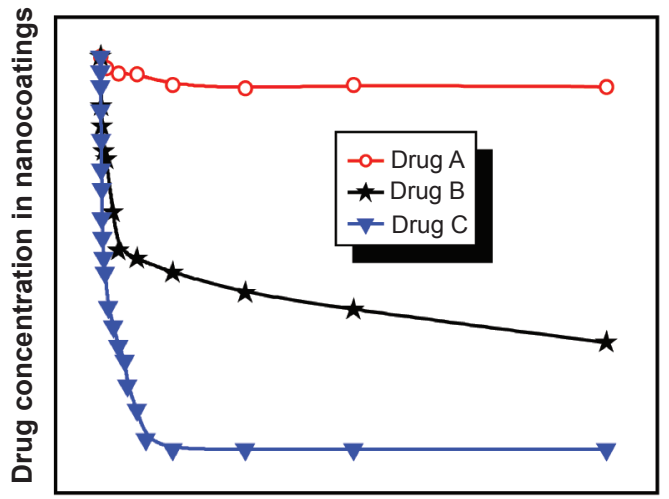

Time

Figure 3 a) Multifunctional polyelectrolyte multilayer nanofilm on a device. $\square$ device, $\rightarrow$ covalently bonded drug $A, \rightarrow$ electrostatically bound drugs $B$ and $C$, respectively, where drug $B$ is deposited as a coating component and drug $C$ is electrostatically adsorbed after film preparation. b) The three drugs present three different drug release patterns in one device system, ie, constant concentration (drug A), sustained release (drug B), and burst release (drug C). This enables the device to have multiple functions such as inhibiting bacterial colonization (eg, drug A is covalently bonded gentamicin) and promoting bone healing (eg, drugs B and/or C are electrostatically bound bone morphogenetic proteins).

recommendations expressed in this material are those of the authors and do not necessarily reflect the views of the funding agencies. We also thank Suzanne Smith for proofreading the manuscript.

\section{References}

1. Decher G. Fuzzy nanoassemblies: Toward layered polymeric multicomposites. Science. 1997;277:1232-1237.

2. Hammond PT. Form and function in multilayer assembly: New applications at the nanoscale. Adv Mater. 2004;16:1271-1293.

3. Baron R, Willner B, Willner I. Biomolecule-nanoparticle hybrids as functional units for nanobiotechnology. Chem Commun. 2007;28: 323-332.

4. Sahoo SK, Parveen S, Panda JJ. The present and future of nanotechnology in human health care. Nanomedicine. 2007;3:20-31.

5. Picart C, Elkaim R, Richert L, et al. Primary cell adhesion on RGDfunctionalized and covalently crosslinked thin polyelectrolyte multilayer films. Adv Funct Mater. 2005;15:83-94.

6. Likibi F, Jiang BB, Li B. Biomimetic nanocoating promotes osteoblast cell adhesion on biomedical implants. J Mater Res. 2008;23(12): 3222-3228

7. Richert L, Lavalle P, Payan E, et al. Layer by layer buildup of polysaccharide films: Physical chemistry and cellular adhesion aspects. Langmuir. 2004b;20:448-458.

8. Richert L, Schneider A, Vautier D, et al. Imaging cell interactions with native and crosslinked polyelectrolyte multilayers. Cell Biochem Biophys. 2006;44:273-285.

9. Vazquez CP, Boudou T, Dulong V, et al. Variation of polyelectrolyte film stiffness by photo-cross-linking: A new way to control cell adhesion. Langmuir. 2009;25:3556-3563.

10. Schneider A, Richert L, Francius G, et al. Elasticity, biodegradability and cell adhesive properties of chitosan/hyaluronan multilayer films. Biomed Mater. 2007;2:45-51.

11. Richert L, Boulmedais F, Lavalle P, et al. Improvement of stability and cell adhesion properties of polyelectrolyte multilayer films by chemical cross-linking. Biomacromolecules. 2004a;5:284-294.

12. Thierry B, Kujawa P, Tkaczyk C, et al. Delivery platform for hydrophobic drugs: Prodrug approach combined with self-assembled multilayers. J Am Chem Soc. 2005;127:1626-1627.
13. Crouzier T, Ren K, Nicolas C, et al. Layer-by-layer films as a biomimetic reservoir for rhBMP-2 delivery: Controlled differentiation of myoblasts to osteoblasts. Small. 2009;5:598-608.

14. Fu J, Ji J, Yuan W, Shen J. Construction of anti-adhesive and antibacterial multilayer films via layer-by-layer assembly of heparin and chitosan. Biomaterials. 2005;26:6684-6692.

15. Fu JH, Ji J, Fan DZ, et al. Construction of antibacterial multilayer films containing nanosilver via layer-by-layer assembly of heparin and chitosan-silver ions complex. J Biomed Mater Res. 2006;79A: 665-674.

16. Yu DG, Lin WC, Yang MC. Surface modification of poly(L-lactic acid) membrane via layer-by-layer assembly of silver nanoparticle-embedded polyelectrolyte multilayer. Biocon Chem. 2009;18:1521-1529.

17. Jiang BB, Li B. Tunable drug incorporation and release from polypeptide multilayer nanofilms. Int J Nanomedicine. 2009;4:37-53.

18. Li B, Jiang BB, Boyce BM, Lindsey BA. Multilayer polypeptide nanoscale coatings for the prevention of biomedical device associated infections. Biomaterials. 2009;30:2552-2558.

19. Zhong Y, Li B, Haynie DT. Fine tuning of physical properties of designed polypeptide multilayer films by control of $\mathrm{pH}$. Biotechnol Prog. 2006;22(1):126-132.

20. Qi B, Tong X, Zhao Y. Layer-by-layer assembly of two different polymer micelles with polycation and polyanion coronas. Macromolecules. 2006;39:5714-5719.

21. Zhong Y, Whittington CF, Haynie DT. Stimulated release of small molecules from polyelectrolyte multilayer nanocoatings. Chem Commun. 2007; 14:1415-1417.

22. Quinn JF, Caruso F. Thermoresponsive nanoassemblies: Layer-bylayer assembly of hydrophilic-hydrophobic alternating copolymers. Macromolecules. 2005;38:3414-3419.

23. Wang L, Wang X, Xu MF, et al. Layer-by-layer assembled microgel films with high loading capacity: Reversible loading and release of dyes and nanoparticles. Langmuir. 2008;24:1902-1909.

24. Berg MC, Zhai L, Cohen RE, Rubner MF. Controlled drug release from porous polyelectrolyte multilayers. Biomacromolecules. 2006;7: 357-364.

25. Scranton AB, Rangarajan B, Klier J. Biomedical applications of polyelectrolytes. Adv Polym Sci. 1995;122:1-54.

26. Chluba J, Voegel JC, Decher G, et al. Peptide hormone covalently bound to polyelectrolytes and embedded into multilayer architectures conserving full biological activity. Biomacromolecules. 2001;2:800-805. 
27. Jessel N, Atalar F, Lavalle P, et al. Bioactive coatings based on a polyelectrolyte multilayer architecture functionalized by embedded proteins. Adv Mater. 2003;15:692-695.

28. Cai KY, Rechtenbach A, Hao JY. Polysaccharide-protein surface modification of titanium via a layer-by-layer technique: Characterization and cell behavior aspects. Biomaterials. 2005;26:5960-5971.

29. Schultz P, Vautier D, Richert L, et al. Polyelectrolyte multilayers functionalized by a synthetic analogue of an anti-inflammatory peptide, alpha-MSH, for coating a tracheal prosthesis. Biomaterials. 2005;26:2621-2630.

30. Lu ZZ, Wu J, Sun TM, et al. Biodegradable polycation and plasmid DNA multilayer film for prolonged gene delivery to mouse osteoblasts. Biomaterial. 2008;29:733-741.

31. Werner S, Huck O, Frisch B, et al. The effect of microstructured surfaces and laminin-derived peptide coatings on soft tissue interactions with titanium dental implants. Biomaterials. 2009;30:2291-2301.

32. Chung AJ, Rubner MF. Methods of loading and releasing low molecular weight cationic molecules in weak polyelectrolytes multilayer films. Langmuir. 2002;18:1176-1183.

33. Wood KC, Boedicker JQ, Lynn DM, et al. Tunable drug release from hydrolytically degradation layer-by-layer thin films. Langmuir. 2005;21:1603-1609.
34. Sukhishvili SA, Granick S. Layered, erasable, ultrathin polymer films. $J$ Am Chem Soc. 2000;122:9550-9551.

35. Dubas ST, Farhat TR, Schlenoff JB. Multiple membranes from "true" polyelectrolyte multilayers. J Am Chem Soc. 2001;123:5368-5369.

36. Quinn JF, Caruso F. Facile tailoring of film morphology and release properties using layer-by-layer assembly of thermoresponsive materials. Langmuir. 2004;20:20-22.

37. Kim BS, Park SW, Hammond PT. Hydrogen-bonding layer-by-layerassembled biodegradable polymeric micelles as drug delivery vehicles from surfaces. ACS Nano. 2008;2:386-392.

38. Serizawa T, Yamaguchi M, Akashi M. Alternating bioactivity of polymeric layer-by-layer assemblies: Anticoagulation vs procoagulation of human blood. Biomacromolecules. 2002;3:724-731.

39. Serizawa T, Yamaguchi M, Akashi M. Time-controlled desorption of ultrathin polymer films triggered by enzymatic degradation. Angew Chem Int Ed. 2003;42:1115-1118.

40. Etienne O, Gasnier C, Taddei C, et al. Antifungal coating by biofunctionalized polyelectrolyte multilayered films. Biomaterials. 2005;26:6704-6712.
Nanotechnology, Science and Applications

\section{Publish your work in this journal}

Nanotechnology, Science and Applications is an international, peerreviewed, open access journal that focuses on the science of nanotechnology in a wide range of industrial and academic applications. It is characterized by the rapid reporting across all sectors, including engineering, optics, bio-medicine, cosmetics, textiles, resource

\section{Dovepress}

sustainability and science. Applied research into nano-materials, particles, nano-structures and fabrication, diagnostics and analytics, drug delivery and toxicology constitute the primary direction of the journal. The manuscript management system is completely online and includes a very quick and fair peer-review system, which is all easy to use. 